\title{
\begin{tabular}{l|} 
EXPERT \\
REVIEWS
\end{tabular}
}

\section{The psychological and social consequences of miscarriage}

\author{
Expert Rev. Obstet. Gynecol. 6(3), xxx-xxx (2011)
}

\author{
Olga BA van den Akker \\ School of Health \& Social Sciences, \\ Middlesex University, The Town Hall, \\ The Burroughs, Hendon, London, \\ NW4 $4 B T$, UK \\ Tel.: +4402084116953 \\ Fax: +4402084114259 \\ o.vandenakker@mdx.ac.uk
}

This article summarizes the research detailing factors relevant to the identification and estimation of medically defined miscarriages, and describes research highlighting the psychological effects and individual social context of miscarriage. Specifically, issues of culture and social psychological interpretations of miscarriage are discussed in this article, and suggestions for improvement of follow-up care are discussed. The research reviewed shows there is a need for immediate and longer term recognition of the psychosocial effects of miscarriage and support for women, particularly those not supported within their social network, and emphasizes the need to be culturally aware when addressing health policy and healthcare needs of different populations undergoing similar reproductive losses.

KEYwORDs: follow-up care $\bullet$ miscarriage $\bullet$ pregnancy $\bullet$ psychological effects $\bullet$ support $\bullet$ trauma

Reproduction is a fundamental consequence of unprotected natural sexual activity, which is the desired outcome for the majority of the population. Pregnancy for the woman and the impending expansion of the self, the couple or the family is a milestone for every man and woman of reproductive age. Pregnancy also defines a woman as reproductively capable and provides her with a new identity of mother. Miscarriage affects $15-20 \%$ of all clinically recognized pregnancies, it is the most common complication of pregnancy and occurs in approximately one-third of all women [1]. Its impact can be devastating upon the individual and can last a long time [2]. The term spontaneous abortion is a misnomer for miscarriage, and is offensive to women who miscarried and their partners, causing further unnecessary distress to their already compromised emotional states. The reported incidence is likely to be higher, because not all pregnancies are clinically confirmed [3], and not all women seek treatment following miscarriage [4]. Miscarriage can involve unexpected pain and significant blood loss and when this happens, the pain and bleeding may make a miscarrying woman sensitive to the care she receives from her healthcare providers [5]. In Northern Ireland, when a miscarriage classification is used, no formal record of death is issued and usually no opportunity for burial is given [6]. There is some debate as to whether the emotional response to a miscarriage is experienced more strongly immediately following the loss compared with weeks, months or years later, and whether a late loss is experienced stronger emotionally than an early loss.

Fetal death in early pregnancy up to the gestational age of approximately 22 weeks is referred to as a miscarriage, with any Fetal death after the 23 rd week being considered a stillbirth - the exact cutoff point varies by country. For example, Australian policy defines a miscarriage as a spontaneous loss of pregnancy before the 20th week; the UK, 24th week [7]; and in some, but not all, American States before the 28th week [8]. It is useful to have some international agreement on the defining time scales for miscarriages and stillbirths, because it is not easy to reconcile a miscarriage at 10 weeks gestation, before visible and tactile evidence of a pregnancy is apparent, compared with a loss at 25 weeks, when the carrying mother is usually visibly pregnant and she has felt the baby move. The 10 -week loss will not be noticed by the social and workplace environment but the 25 -week loss is very noticeable to all who know the woman. The 10-week loss is therefore not accompanied by the social awareness, social support and healthcare support that are afforded to a later pregnancy loss. Stillbirths, or late pregnancy losses, are accompanied by a stillbirth certificate, and the opportunity to have a naming and burial for the baby.

Miscarriage can also involve experiencing distressing physical symptoms, particularly if medical intervention, (evacuation of the foetus) was used and continued heavy bleeding occurs'. There is no evidence that different methods used 
to remove the foetus result in different psychological reactions. For example, Nielsen $e t$ al. reported similar grief reactions in women undergoing $\mathrm{D} \& \mathrm{C}$ versus expectant management [9]. Both miscarriage and stillbirth may be accompanied by an awareness of other medical or genetic factors, which may all contribute to the psychological distress experienced. It is important to note, however, that in some cases a miscarriage marks the beginning of something positive, such as, for example, a relief of an unwanted pregnancy $[10,11]$. The reactions therefore vary enormously from person to person depending on individual differences, and the personal significance of the loss to the individuals concerned.

The fact that miscarriage statistics are reported as approximate is in part because many miscarriages occur at home and are not reported, in addition many are biochemical and unknown to the woman. Furthermore, miscarriages can be presented in different ways. For example, statistics of miscarriages have been reported for different age groups, as shown, for example, in an Australian study of 9145 young women, that compares the incidence of miscarriages across young women in two age groups (FIGURE 1) [12].

However, a reproductive life-span view of miscarriages shows an even steeper incremental rise in miscarriages with increasing age, as shown in Figure 2 .

Miscarriages have also been reported according to physical characteristics of the event. For example, a differentiation has been made between missed miscarriages, incomplete miscarriages and complete miscarriages, listed in the follwing:

- Missed miscarriage refers to a fetus that is not expelled by the woman, and the missed miscarriage may not be noticed for several weeks. No pregnancy symptoms may be present, but they may have elevated hormone levels;

- Incomplete miscarriage refers to the partial or incomplete expulsion of the fetal or pregnancy tissue. Here, parts of the fetus, amniotic sac and/or placenta may be retained, whilst part of these may be expelled. The cervix is likely to be open and cramping, and bleeding is common;

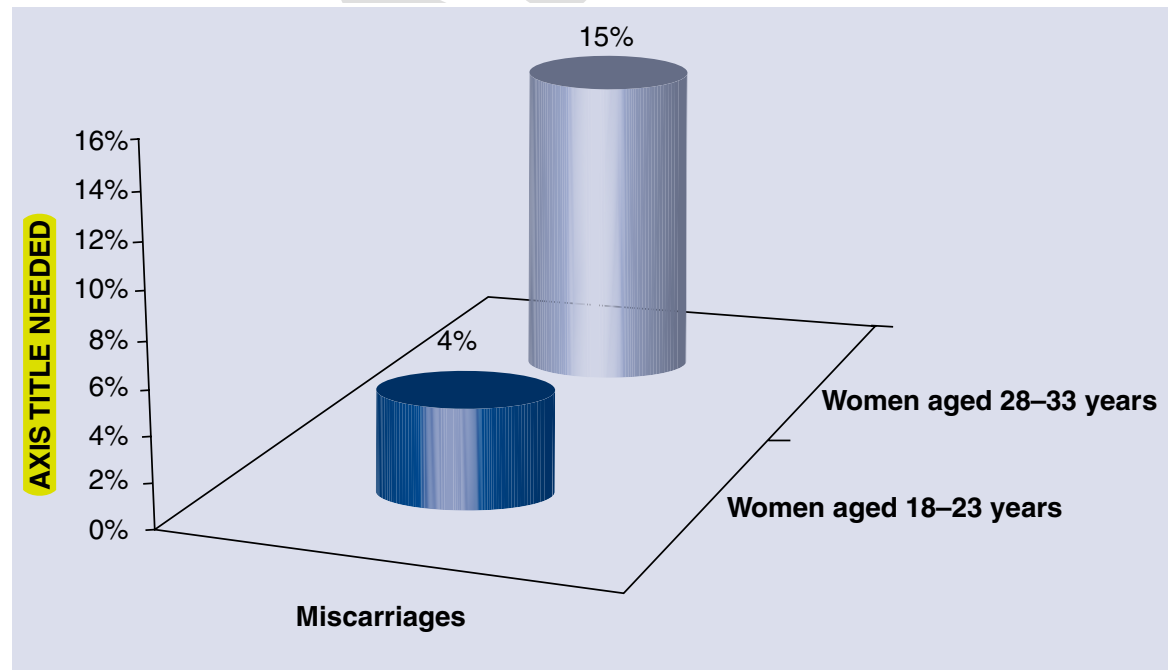

Figure 1. Showing incidence of miscarriages across young women in two age groups.
- Complete miscarriage refers to the complete expulsion or delivery of a nonviable fetus. Here, all fetal, amniotic and placental tissue is expelled and the cervix is closed;

Missed and incomplete miscarriages require medical intervention such as a $\mathrm{D} \& \mathrm{C}$, if the tissue is not eventually expelled in its entirety naturally.

The Physical signs of a miscarriage include:

- Back pain ranging from mild to severe;

- The woman has lost weight (in later miscarriages, or when marked by significant grief reactions);

- The appearance of pink-colored mucus;

- Rhythmic contractions ranging from mild to severe;

- Tissue clots and bleeding;

- Decreasing signs of pregnancy.

Although there is a varying amount of bleeding, cramping, backache and abdominal pain involved in miscarriages, the psychological and social effects may be considerably more severe and longer lasting. The miscarriage symbolizes a number of areas that define an individual across societies and cultures. For example, a miscarriage in terms of a person's identity signifies disappointments and challenges of:

- Motherhood identity and role;

- Fatherhood identity and role;

- Family identity and role;

- Death of a baby;

- Social and normative deviations;

- Stigma and isolation;

- The death of a dream often held since childhood.

This article outlines the grief and coping processes that follow miscarriage in a number of cultural and social contexts across societies, contributing to the psychological distress experienced by those affected. Since miscarriages are a common pregnancy complication, the psychosocial costs associated with miscarriages need be recognized and targeted for appropriate care.

\section{Risk factors predisposing women to miscarry}

Although the evidence is conflicting [13], the risk of miscarriage is not the same for women of different ages, as shown earlier, with estimates ranging from 9 to $75 \%$ even within studies [14]. Maconochie et al. reported that paternal and maternal age, previous miscarriages, terminations, 
infertility, assisted conception, low BMI prepregnancy, regular alcohol consumption, stress and changing partner are independent risk factors for miscarriages [15]. Since delayed childbearing is becoming more common in all countries where controlled conception is available, it is likely that this delay will be accompanied by an increase in (reported and unreported) miscarriages. In addition to first-time miscarriages, recurring miscarriage,s although less common with estimates between 1 and $2 \%$ of women, affect women who have three or more consecutive miscarriages [16], although others argue two or more miscarriages constitute recurrent miscarriage, increasing the proportion to $5 \%[17]$. When women present with recurrent miscarriages, they tend to be referred for investigations to determine the cause of the problem.

Despite the disagreement about a universally adhered to definition of recurrent miscarriage, the incidence is more than is expected by chance alone, suggesting specific reasons should be responsible for them, although to date no effective treatment exists, as evidenced, for example, in a recent randomized controlled trial (RCT) [18]. Nevertheless, age [19] and number of previous miscarriages [20] are independent risk factors, although they are of course interlinked, for example, older women are more likely to miscarry than younger women, and the more miscarriages a woman has had, the more likely it is that she will have more. Chromosomal abnormalities and particular autoimmune issues such as antiphospholipid antibodies are more likely to account for recurrent miscarriages than embryonic abnormalities. Anatomical abnormalities such as major uterine irregularities, a weak or incompetent cervix, and indirectly, polycystic ovaries (causing high LH levels) are also thought to be responsible for recurrent miscarriages. Numerous other factors, such as infective, endocrine, immune, thrombophilias and unknown etiological factors are believed to be responsible for some recurrent miscarriages, but the evidence is equivocal [201]. The psychological effects of recurrent miscarriage, including being told to wait until the next time before investigations are carried out, can be devastating [21]. Research has shown that being informed that no treatment is available, or no known cause has been identified is problematic [21-23]. Whether it is beneficial for clinicians to advise women who miscarried to wait for a new pregnancy is not certain. According to Klier et al. [24], it may be more beneficial for the prospective parents to be in control of their own decisions, since other evidence suggests that feeling in control and having a choice poses a relief to some patients [25].

Psychological research has postulated that psychological factors such as aspects of personality [26] or depression can be causally related to miscarriages $[27,28]$, although others have questioned its significance $[29,30]$. Nevertheless, a recent meta-analysis has demonstrated that $18 \%$ of women experience depressed mood during pregnancy, and approximately $13 \%$ of these meet the Diagnostic and Statistical Manual of Mental Disorders (DSM-IV) [31] diagnostic criteria for a major depressive disorder [31]. Other more recent research has reported increased pregnancy-specific anxiety and depression in newly miscarrying women who had miscarried previously and in those who had not experienced a previous pregnancy loss [2,7]. It is possible that psychological stress or anxiety can affect adequate immune system functioning, leading to pregnancy loss [33], and therefore needs to remain a key factor in future research. Furthermore, sociodemographic risk factors including, marital status, parity and lifestyle or behavioral factors such as being a smoker or ex smoker, high BMI, being a noncontraceptive user and having a lower education and socioeconomic status have also been identified in a large Australian study [34]. These studies suggest that psychological and behavioral variables cannot be ignored in future research and practice of reproductive loss.

\section{The psychological effects of miscarriage}

Within miscarrying women, reactions tend to be varied. A number of risk factors predisposing women to experience significant psychological distress or morbidity following miscarriage have 
also been identified. For example, a history of psychiatric illness, childlessness and a lack of partner or other social support, ambivalence towards the fetus, as well as previous pregnancy loss, are likely to increase the chances of severe psychological distress. A study of suicides in Finland identified a significantly higher mean annual suicide rate in women who had miscarried in the year prior to their suicide (18.1 out of 100,000$)$ compared with women who had delivered a baby $(5.9$ out of 100,000) [35]. However, these reactions tend to be extreme.

Nevertheless, although in miscarriage women and men are not always in a position to mourn a fully grown baby, the miscarriage represents the loss of the opportunity to give birth to a live baby. Critically, pregnancy loss robs women and men of the opportunity to raise the child, become a parent, and form a new identity as a parent. The loss following a miscarriage is hidden and relatively unquestioned by healthcare staff, friends and family and at the extreme end can develop into symptoms of trauma [36]. The consequences of facing this traumatic experience alone in relative isolation can have severe psychological consequences. Gerber-Epstein, Leichtentritt and Benyamini described the need to recognize that the impact of the miscarriage may be proportional to the desire for the pregnancy [37]. The social and cultural environment tends to have near universal pronatal expectations, and ignores the need to acknowledge the failed pregnancy and mourning [38]. Research has also demonstrated the need for women in some cultures to mourn and cope with their loss in isolation because the societal ramifications of pregnancy loss are harsh upon the women experiencing them [39]. Stigma and accusations from the wider social network therefore can impose a double blow on women already compromised.

Awareness of the severity of the physical effects of miscarriage are increasingly recognized as demonstrated by, for example, a USA report of $>60$ maternal deaths in a 10 -year period that are associated with miscarriages [40], and data showing fertility problems increasing with increasing numbers of miscarriages [41].

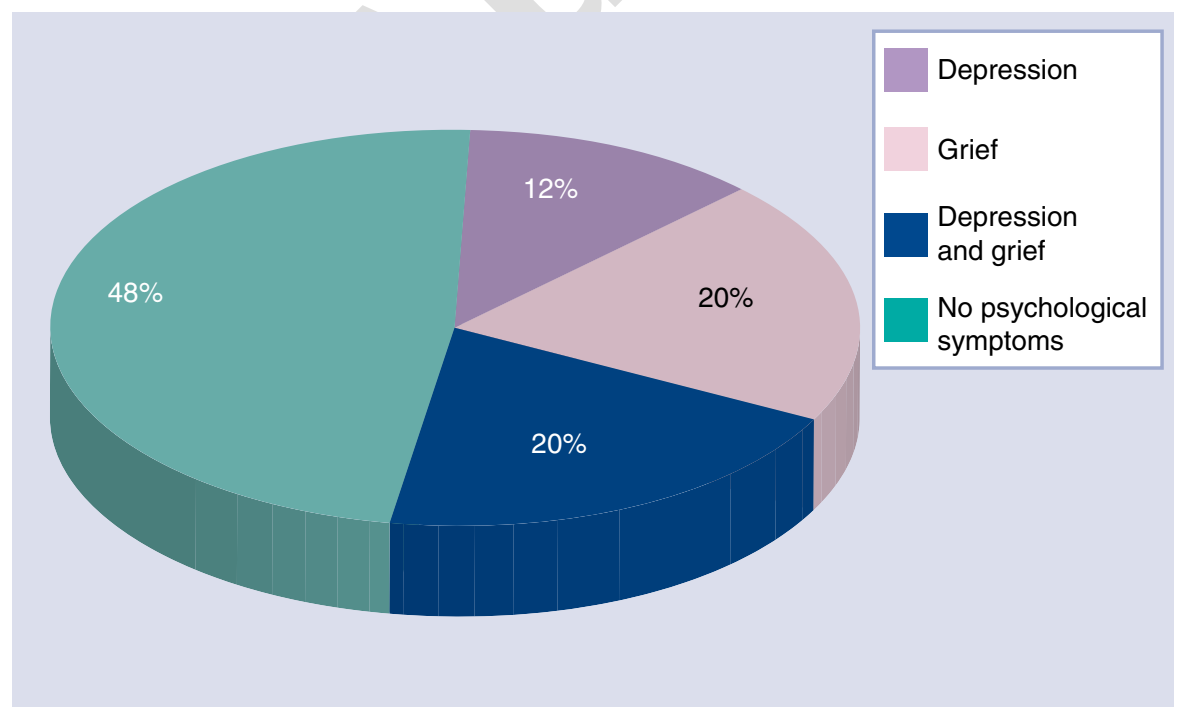

Figure 3. Showing different psychological reactions of women immediately post miscarriage.
The severity of the psychological effects of miscarriages has also been reported in women and to a lesser extent in their partners [37] and siblings [42]. For example, increased anxiety lasting up to 4 months after the miscarriage [43], depression [44] and grief reactions have been reported in women and men immediately after miscarriages [45,46]. DSM-IV includes grief and sadness within a description of major depressive episodes in conjunction with symptoms such as loss of appetite, weight loss, guilt, insomnia and morbid thoughts [31]. Reports of strain on the couple's relationship following the miscarriage [47], post-traumatic stress disorder $[48,49]$, obsessive-compulsive disorders [50], and panic disorder [51] have also been described. A number of these psychological effects are clinically significant syndromes and disorders [52,53] requiring adequate identification and treatment. Routine follow up to identify significant psychopathology following miscarriage is not universally available.

However, identification and assessment of clinically effective states may be difficult to establish in practice, particularly since research has shown that, even in the psychological morbidity literature, no consistency in the use of diagnostic criteria is evident. In a review article, Lok and Neugebauer reported that between 20 and $55 \%$ of women in the studies they looked at reported elevated levels of depression in the short term after miscarriage, but only five studies used diagnostic criteria [13]. Rowlands identified 33 studies which assessed the incidence of depression and although use of comparison and control groups differed and timescales and measures were not constant, all studies showed increases in depression in miscarrying women [54]. In another study, depression was reported up to 1 year following the miscarriage [44]. Conway and Russel [45] comment that miscarriages are not usually recognized as a loss similar to bereavement, and that grieving is not understood following a miscarriage. However, grieving, even if the loss is not perceived as a bereavement or loss of a potential child, is nevertheless worthy of recognition, screening and counseling or other treatment. The lack of social support for women who have miscarried in a vacuum without rites, rituals or acknowledgements of, for example, the 'anniversary effect' on the date of miscarriage or estimated delivery date [55], may prevent closure of the life event for many women.

Research has shown that at any stage, a miscarriage can result in symptoms of sadness, yearning for the lost fetus/child, a need to talk about the loss and search for meaning and explanation of the loss [56,57]. Figure 3 shows the proportion of women reporting grief, depression, combined grief and depression or no elevated emotional distress in the study by Beutel et al., using consecutively miscarrying women [56]. These authors demonstrated longer lasting psychological and social symptomatology (at 6 and 12 months postmiscarriage) in women who were 
identified with initial depressive states following the miscarriage, suggesting they are at increased risk. However, although the samples were relatively small, the study also showed that nearly half their sample reported no adverse psychological symptomatology. Grief intensity and duration is reported to be the same for early and late miscarriage [8,58-60], but tends to be longer for perinatal death $[61,62]$. Few studies have explicitly differentiated the effects of multiple miscarriages on women. Klock $e t a l$. reported high state-anxiety scores and relatively high enduring trait-anxiety in women who had an average of three miscarriages [63]. Anxiety and depression have also been monitored in a longitudinal study which reported $49 \%$ of clinically significant cases for anxiety 5 weeks after the miscarriage, which remained evident for $27 \%$ of the women 17 weeks after the loss [64]. This suggests that clinically significant psychological morbidity remains a serious and potentially debilitating concern for a significant number of women for as long as 4 months following the (repeated) miscarriage. Impaired quality of life and economic effects for those affected by recurrent miscarriage is therefore likely.

There is less agreement in the literature studying differences between the psychological effects of miscarriage on the miscarrying women's male partners. Johnson and Baker reported increases in anxiety and depression in men immediately after the miscarriage, which diminished by 1 year post miscarriage [65]. In a study of couples who had experienced a miscarriage, men reported significantly lower anxiety scores than the women immediately following the miscarriage, although the men's scores were higher than community controls [66]. Daly and Harte, on the other hand, reported morbid levels of anxiety in $36 \%$ of male partners 6 weeks following miscarriage [67]. A 2-year follow-up study of miscarrying women and their male partners reported that less grief, fear, loneliness and depression was reported in the men compard with the women [68]. Johnson and Puddifoot reported greater despair in men who had witnessed the ultrasound scan prior to the miscarriage, than those who had not experienced the visual information of the fetus [69]. One other qualitative study reported higher grief responses in men than women immediately after and 4 months after the miscarriage [45]. Coping with miscarriage was also reported to be different between men and women, with both seeking social support, however, women were more likely to seek spiritual support, whereas men tended to worry and try to ignore the situation [70].

\section{Recognition of miscarriage}

Cultural and religious differences in the recognition of miscarriage and subsequent coping with and grieving processes of the lost pregnancy, the fetus or baby (following miscarriage) have been identified in early psychosocial research studying the effects of miscarriages [71]. Hsu, Tseng and Kuo reported on incomplete grieving processes in a study group of Taiwanese mothers who had experienced a perinatal death, but because of cultural norms and taboos, were prevented from talking about the death or taking part in death-related rituals [72]. African-American women, however, believe that spiritual or religious activities can aid coping with miscarriage [73], as did McGreal, Evans and Burrows's Caucasian women [70]. More research is needed to determine the influences of culture, traditions and religion on coping with miscarriage, but it is likely to be relevant in all of today's multicultural societys' healthcare systems. The clinical implications of the effects of miscarriage and the associated public health effects are substantial.

Culturally determined reactions may influence the experiences of miscarriage in women even when they have moved outside of the culture of origin. For example, Mexican or Hispanic/ Latino populations have increased in the USA since the 1970s. Interestingly, the birthweights of first-generation Mexican women, who were born in Mexico but moved to the USA, are reportedly better than those of second-generation US women of Mexican descent [74]. These data go against what is known about usual risk factors, which is that first-generation immigrants tend to be older, of lower socioeconomic status and they tend to receive less optimal healthcare [75], suggesting the culture of origin might be protective in some ways. However, no effects for miscarriage were obtained, indicating generational differences or acculturation is not a factor in miscarriage. Acculturation refers to behaviors that are specific to the culture of origin or lifestyle associated with that culture - which can protect against adverse pregnancy outcomes - and which continues to influence individuals in their new environment, whilst adapting some new cultural norms. Sefton qualitatively explored the long-term reactions to miscarriage of 14 young adolescent Latino women, and reported a lack of specific reaction attributable to culture of origin in Latino women with reactions ranging instead from minimal to long-term unresolved grief [76].

Although cross-cultural research in this area is rare, psychosocial research has shown women need to understand their loss and to be given a chance to mourn their lost pregnancy [77]. The loss, grief, sadness, fear, guilt and alarm reactions in women who miscarried and the intensity of these feelings have been described by Abboud and Liamputtong, and these varied depending on the personal circumstances and the reactions of others to their miscarriage [78]. In some developing countries the cultural ethos dictates a stigma associated with reproductive loss and a lack of empowerment (access to education, healthcare and an independent income) for many women, who therefore are prohibited from expressing their emotional needs for fear of the social consequences [39,72]. The National Women's Health Study [79] qualitatively describes the personal stories of women from a nationally representative, population based, postal survey of the reproductive history of adult women in the UK, through an open-ended section for additional comments. The thematic analysis carried out on the open ended responses by Simmons et al., showed there were opposing tensions between the need for a medical explanation for the loss and at the same time a perception of inappropriate mediatization of the experience of the miscarriage [80]. There was an emphasis on the experience of miscarriage as an individual and serious experience as opposed to the medical view of this as a routine complication. Women also noted the effect of the healthcare professional's behaviors and how these affected their own interpretations of 


\section{Table 1. Showing domains for future post-} miscarriage service delivery.

Staff care

Information

Risk assessment

the miscarriage event.

\section{Psychological management of women following miscarriage}

Since the majority of the research evidence suggests the long-term effects for some women may be severe, it is critical that treatment does not stop immediately after the confirmation of miscarriage or associated evacuation of the fetus. Bergner et al. [81] conducted a longitudinal quantitative analysis of 232 women with first-trimester pregnancy loss [81]. They found that maladaptive or less useful coping strategies and psychosocial risk factors predicted increased depression at 7 months postmiscarriage and this carried on into a new pregnancy. Psychopathology in a subsequent pregnancy was also further predicted by a pattern of anxious grieving. Healthcare providers should monitor support isolation in women following miscarriage, since evidence unequivocally suggests women who are insufficiently supported socially from their partners or from their social network, develop stronger grief reactions than those in supportive relationships [82-84]. It is therefore unsurprising that research has associated martial satisfaction with decreased psychopathology following miscarriage $[85,86]$.

Since miscarriage is largely diagnosed and treated with by the medical, nursing and midwifery professions, they may be less familiar with psychological and psychiatric assessments, and hence fail to identify psychological and psychiatric morbidity in the populations they are responsible for. To date, there is a lack of unified psychological assessment available measuring psychological morbidity in women and men postmiscarriage. Nevertheless, since anxiety and depression feature in the majority of women and men in the studies reported in this article, a number of generic measures such as the General Health Questionnaire [87] or the Hospital Anxiety and Depression [88] scales, coupled with questions of grief reactions and symptoms of trauma, or the Perinatal Grief Scale [62], could provide some insight into the psychological needs of these couples.

According to Nikcevic, Tunkel and Nicolaides the majority of women who were asked, believed they would benefit from follow-up care [89]. In practice, only a minority actually receive any postmiscarriage care, and satisfaction with healthcare services was low [90,91]. Research in the UK in the mid-1990s repeatedly demonstrated the need for information and attitudes of medical staff negatively affecting the experience of the management of miscarriage [92-94]. More recently, Sejourn, Callahan and Chabrol reported on an internet-based survey of over 300 women who had previously miscarried [95]. They found that the majority of women believed they would have benefited from support following their miscarriage. Information regarding causes of the miscarriage were particularly desired, as they believed they were generally poorly informed about the medical aspects and psychological consequences of their miscarriage. Stratton and Lloyd assessed the literature for the availability of evidence-based hospital guidelines for medical and psychosocial services during and following miscarriage [96]. They found little evidence of effective follow up of women who miscarried after discharge from hospital, and propose a number of foci for future improved miscarriage and postmiscarriage service delivery (TABLE $\mathbf{1}$ ).

It is timely for healthcare professionals to develop a deeper insight into the psychological and social effects of miscarriage and accept it for a real and significant loss which should be recognized and supported. Current evidence outlined in a recent review has shown that those who have experienced a miscarriage report low levels of satisfaction if the healthcare providers' attitudes were perceived to be negative, if contact with healthcare involved 'entering and exiting a medical facility quickly', or if information was insufficient and follow-up care did not consider their psychological needs [97]. Medical and allied healthcare professionals could refer women to a mental health professional for a more thorough evaluation. For example, psychologists specializing in women's health may be ideally placed to assess and subsequently assist women who experience reproductive loss. Supportive treatment facilitating adaptive coping strategies could be offered [81], and may enhance a speedier recovery from the loss.

There is some evidence that counseling programs for couples who experienced neonatal death and stillbirth is effective [98]. No such counseling programs exist for people who have experienced miscarriage. One RCT attempted to elucidate the efficacy of early ( 4 months postmiscarriage) or delayed (1 year following miscarriage) counseling care versus an early or delayed control condition, and reported a specific caring effect in improvement in overall emotional disturbance, and at 1 year, both the control and intervention arms resulted in increased self esteem and decreased psychological symptoms [99]. A small-scale RCT, assessing the effectiveness of interpersonal counseling versus usual care also indicated a significant reduction in negative psychological effects in the counseling group compared with usual care group [100]. A number of qualitative studies further support the need for emotional support and follow-up [5,101-103]. Women in Adolfsson et al.'s study felt vulnerable and abandoned by healthcare professionals and neglected by their close social networks [5]. Over the last decade, Early Pregnancy Assessment Units in the UK have shown some promising results for the welfare of women who miscarried [104] as have follow-up care units for women suffering from pregnancy loss [105,106]. However, these initiatives are not universal, and across the globe women are unhappy with the healthcare received following miscarriage. Within reproductive health practice, geneticists and social scientists have sought to include cross-cultural issues when counseling people from ethnic minorities who have yet to acculturate or assimilate into the normative culture of residence [107,108], as well as women with physical disabilities or mental retardation, who are also making reproductive decisions. There is a need for effective strategies to address the unique needs posed by all individuals seeking reproductive healthcare, particularly if they are already vulnerable. For 
healthcare systems to rely on (often informal) support groups and/ or online forums, to talk through their experiences [6] is healthcare's missed opportunity to support the essential psychological needs of millions of women and men. The gap between health policies across the world - which focus on the medical aspects of reproductive care - and the lived experiences of the women and men, needs to close universally [109].

\section{Expert commentary}

The vast majority of the reproductive population of women and men are insufficiently prepared for a miscarriage, and the findings reported in this article show that many women and men experience some negative reactions, which may include reactions of grieving. For some women, the symptoms experienced following miscarriage are severe enough to mimic symptoms of trauma and other psychopathology which may last for weeks or months following the miscarriage and carry over into a new pregnancy. The importance of psychological factors directly affecting pregnancy or mediating the effects leading to pregnancy loss continues to be underestimated in clinical practice, despite research extrapolating their importance. Psychological stress or anxiety can affect immune system functioning, which in turn, can lead to a new pregnancy loss. Furthermore, sociodemographic and behavioral risk factors including, marital status, parity, being a smoker or ex smoker, a noncontraceptive user and having a lower education and socioeconomic status have also been shown to be important, and some of these (behavioral factors) can be modified. Assessment of psychological morbidity following miscarriage is ad hoc and appropriate behavioral modification or psychological clinical care is virtually nonexistent. There is an urgent need to re-evaluate the services currently offered within and between countries and support services should be made available to those who need this.

\section{Five-year view}

The last few decades have seen an increase in the recognition that miscarriages can result in significant and lasting psychological distress for many women and for their partners. Research addressing the psychosocial effects of miscarriage in the next 5 years should focus on the perceived losses experienced by the individuals and the couples. The meaning attached to the failed pregnancy, the lost baby, the inability to create a family, the personal attributions of woman/mother, man/father and the stigma associated with the failed pregnancy should also be addressed. Cultural perspectives affect the experience of miscarriage, and within multicultural societies, much can be done to improve our understanding of the losses experienced. Research assessing the effectiveness of clinical practice has recognized the importance of the psychological effects of a previous miscarriage, coping styles and social support as well as the importance of behavioral and lifestyle factors in the etiology of miscarriage.

Critically, pregnant women who have miscarried before are at increased risk of psychiatric morbidity during their subsequent pregnancy, and these effects could impact on the health and wellbeing of the fetus. Stress and anxiety in pregnancy in particular, need to be recognized early and alleviated, ensuring these effects are not translated to the fetus. Intervention post miscarriage needs to ensure the experience of the miscarriage is fully consolidated, so that the fear and stress associated with a repeat outcome in a new pregnancy are diminished. Behavior and lifestyle modification could also contribute to enhancing the chances of a successful pregnancy.

\section{Financial \& competing interests disclosure}

The author has no relevant affiliations or financial involvement with any organization or entity with a financial interest in or financial conflict with the subject matter or materials discussed in the manuscript. This includes employment, consultancies, honoraria, stock ownership or options, expert testimony, grants or patents received or pending, or royalties.

No writing assistance was utilized in the production of this manuscript.

\section{Key issues}

- The incidence of miscarriage is common in women across the world.

- The psychological and social effects of miscarriage are many fold and can be long lasting.

- Severe effects of miscarriage, including trauma, can affect a new pregnancy.

- Stress and anxiety experienced during a subsequent pregnancy can adversely affect the unborn fetus.

- Support post miscarriage need to be evaluated and recognized.

- Future research should establish clear criteria for post miscarriage impaired psychological functioning and distress.

- Efforts should be made to provide support and counseling to women who do not receive this from their usual support networks.

- Cultural, terminological and methodological factors affect recognition of the physical experience of miscarriage and of the psychological and social effects of miscarriage.

- The gap between health policy and the psychosocial practice of the experience need to be addressed.

\section{References}

Papers of special note have been highlighted as:

- of interest

1 Corbet-Owen C, Kruger LM. The health system and emotional care: validating the many meanings of spontaneous pregnancy loss. Fam. Sys. Health 19(4), 411-427 (2001).

2 Bergner A, Beyer R, Burghard F, Klapp F, Rauchfuss M. Pregnancy after early pregnancy loss: a prospective study of anxiety depressive symptomatology and coping. J. Psychosomatic Obstet. Gynecol. 29(2), 105-113 (2008).

3 Freda M, Devine K, Semelsberger C. The lived experience of miscarriage after infertility. MCN Am. J. Matern. Child Nurs. 28(1) 16-23 (2003). 
4 Hemminki E. Treatment of miscarriage: current practice and rationale. Obstet. Gynecol. 91(2), 247-253 (1998).

5 Adolfsson A, Larsson P, Wijma B, Bertero C. Guilt and empyiness: women's experiences of miscarriage. Health Care Women Internat. 25, 543-560 (2004).

6 McCreight BS. Perinatal loss: a qualitative study in Northern Ireland. Omega 57(1), 1-19 (2008).

7 Tsartsara E, Johnson M. Women's experience of care at a specialised miscarriage unit: an interpretative phenomenological study. Clin. Effectiveness Nursing 6(2), 55-65 (2002).

8 Neugebauer R, Kline J, O’Connor P et al. Determinants of depressive symptoms in the early weeks after miscarriage. $A m$. J. Public Health 82(10), 1332-1339 (1992).

9 Nielsen S, Hahlin M, Moller A, Granberg S. Bereavement, grieving and psychological morbidity after first trimester spontaneous abortion: comparing expectant management with surgical evacuation. Hum. Reprod. 11, 1767-1770 (1996).

10 Slade P. Predicting the psychological impact of miscarriage. J. Reprod. Infant Psychol. 12, 5-16 (1994).

11 Lee C, Slade P. Miscarriage as a traumatic event: a review of the literature and new implications for intervention. J. Psychosomatic Res. 40, 235-244 (1996).

12 Herbert D, Lucke J, Dobson A. Pregnancy losses in young Australian women: findings from the Australian Longitudinal Study on Women's Health. Womens Health Issues 19(1), 21-29 (2009).

- Discusses the need to improve our understanding of pregnancy losses in the general population, and shows how age and delayed family formation differentially affect pregnancy maintenance.

13 Lok I, Neugebauer R. Psychological morbidity following miscarriage. Best Pract. Res. Clin. Obstet. Gynaecol. 21(2), 229-247 (2007).

14 Nybo Anderson A, Wohlfahrt J, Christens P, Olsen J, Melbye M. Maternal age and fetal loss: population based register linkage study. BMJ 320 (7251), 1708-1712 (2000).

15 Maconochie N, Doyle P, Prior S, Simmons R. Risk factors for first trimester miscarriage - results from a UK population based case-control study. BJOG 114, 170-186 (2007).
16 Magee P, MacLeod A, Tata P, Regan L. Psychological distress in recurrent miscarriage: the role of prospective thinking and role and goal investment. J. Reprod. Infant Psychol. 21(1), 35-47 (2003).

17 Rai R, Regan L. Recurrent miscarriage. Lancet 368, 601-611 (2006).

18 Kaandorp SP, Goddijn M, van der Post JAM et al. Aspirin plus heparin or aspirin alone in women with recurrent miscarriage. N. England J. Med. 362(17) 1586-1595 (2010).

19 Anderson N, Wohlfahrt AM, Christens J, Olsen Melbye JM. Maternal age and fetal loss: population based register linkage study. BMJ 320, 1708-1712 (2000).

20 Regan L, Braude PR, Trembath PL. Influence of past reproductive performance on risk of spontaneous abortion. BMJ. 299, 541-545 (1989).

21 Clifford K, Rai R, Regan L. Future pregnancy outcome in unexplained recurrent first trimester miscarriage. Hum. Reprod. 12, 387-389 (1997).

22 Liddell HS, Pattison NS, Zanderigo A. Recurrent miscarriage - outcome after supportive care in early pregnancy. Aust. NZ J. Obstet. Gynaecol. 31, 320-322 (1991).

23 Brigham SA, Conlon C, Farquharson RG. A longitudinal study of pregnancy outcome following idiopathic recurrent miscarriage. Hum. Reprod. 14,2868-2871 (1999).

24 Klier CM, Geller PA, Ritscher JB. Affective disorders in the aftermath of miscarriage: a comprehensive review. Arch. Womens Mental Health 5, 129-149 (2002).

25 Cuisinier M, Janssen H, de Graauw C, Bakker S, Hoogduin C. Pregnancy following miscarriage: course of grief and some determining factors. J. Psychosomatic Obstet. Gynecol. 17, 168-174 (1996).

26 Gannon K. Psychological factors in the aetiology and treatment of recurrent miscarriage. A review and critique. J. Reprod. Infant Psychol. 12, 55-64 (1994).

27 Suguira-Ogasawara M, Furukawa AF, Nakano Y, Hori S, Aoki K, Kitamura T. Depression as a potential causal factor in subsequent miscarriage in recurrent spontaneous aborters. Hum. Reprod. 17(10) 2580-2584 (2002).

- Describes the effect of pre-existing depressive mood on subsequent pregnancy loss.

28 Bonari L, Bennett H, Einarson A, Koren G. Risks of untreated depression during pregnancy. Motherisk Update. Clin.
Practice Can. Fam. Physician. 50, 37-39 (2004).

29 Milad MP, Klock SC, Moses S, Chatterton R. Stress and anxiety do not result in pregnancy wastage. Hum. Reprod. 13(8), 2296-2300 (1998).

30 Bergant AM, Reinstagler K, Moncayo H et al Spontaneous abortion and psychosomatics. A prospective study on the impact of psychological factors as a cause of recurrent spontaneous abortion. Hum. Reprod. 12(5), 1106-1110 (1997).

31 American Psychiatric Association, Task Force on DSM-IV. Diagnostic and Statistical Manual of Mental Disorders(DSM-IV) 4th Edition. American Psychiatric Association, Washington DC, USA (1994).

32 Gavin NI, Gaynes BN, Lohr KN, Meltzer-Brody S, Gartlehner G, Swinson T. Perinatal depression: a systematic review of prevalence and incidence. Obstet. Gynecol. 106, 1071-1083 (2005).

33 Arck PC, Rose M, Hertwig K, Hagen E, Hildebrandt M, Klapp BF. Stress and immune mediators in miscarriage. Hum. Reprod. 16(7), 1105-1111 (2001).

34 Rowlands I, Lee C. Correlates of miscarriage among young women in the Australian longitudinal study on women's health. J. Reprod. Infant Psychol. 27(1), 40-53 (2009).

35 Gissler M, Hemminki E, Lonnqvist J. Suicides after pregnancy in Finland, 1987-1994: register linkage study. BMJ 313, 1431-1434 (1996).

36 Layne L. Unhappy endings: a feminist reappraisal of the women's health movement from the vantage of pregnancy loss. Soc. Sci. Med. 56, 1881-1891 (2003).

37 Gerber-Epstein P, Leichtentritt RD, Benyamini $Y$. The experience of miscarriage in first pregnancy: the women's voices. Death Stud. 33, 1-29 (2009).

38 Borg S, Lasker J. When Pregnancy Fails. Bantam Books, NY, USA (1989).

39 Haws RA, Mashasi I, Mrisho M, Schellenberg JA, Darmstadt GL, Winch PJ. "These are not good things for other people to know": how rural Tanzanian women's experiences of pregnancy loss and early neonatal death may impact survey data quality. Soc. Sci. Med. 71, 1764-1772 (2010).

40 Saraiya M, Green C, Berg C, Hopkins F, Koonin L, Atrash H. Spontaneous abortion - related deaths among women in the United States 1981-1991 Obstet. Gynecol. 94(2), 172-176 (1999). 
41 Garcia-Enguidanos A, Calle M, Valero J, Dominguez-Rojas V. Risk factors in miscarriage: a review. Eur. J. Obstet. Gynecol. Reprod. Biol. 102(2), 111-119 (2002).

42 DeFrain J, Millspaugh E, Xie X. The psychosocial effects of miscarriage: implications for health professionals. Families Systems Health 14(3), 331-347 (1996).

43 Geller P, Kerns D, Klier C. Anxiety following miscarriage and the subsequent pregnancy: a review of the literature and future directions. J. Psychosomatic Res. 56, 35-45 (2004).

44 Cumming G, Klein S, Bolsover D, Lee A, Alexander D, Jurgens J. The emotional burden of miscarriage for women and their partners: trajectories of anxiety and depression over 13 months. BJOG 114(9), 1138-1145 (2007).

45 Conway,K, Russel G. Couples grief and experience of support in the aftermath of miscarriage. Brit. J. Med. Psycho. 73, 531-545 (2000).

46 Broen AN, Moum T, Bødtker AS, Ekeberg O. The course of mental health after miscarriage and induced abortion: a longitudinal, five year follow-up study. BMC Med. 12(3), 18 (2004).

47 Sperry P, Sperry L. The family experience of loss associated with miscarriage and ectopic pregnancy. Family J. Counsel. Ther. Couples 12(4), 401-404 (2004).

48 Bowles SV, Bernard RS, Epperly T, Woodward S, Ginzburg K, Folen R. Traumatic stress disorders following first trimester spontaneous abortion. J. Family Prac. 55(11) 969-973 (2006).

49 Engelhart IM, van den Hout MA, Amtz A. Post traumatic stress disorder after pregnancy loss. Gen. Hosp. Psychiatry 23, 62-66 (2001).

- Prospective longitudinal study emphasizing the occurrence in some women of the extreme psychological states such as post traumatic stress disorder following miscarriage.

50 Neziroglu F, Anemone R, Yaryura-Tobias J. Onset of obsessive-compulsive disorder in pregnancy. Am. J. Psychiat. 149, 947-950 (1992).

51 Klein D, Skrobala A, Garfinkel R. Preliminary look at the effects of pregnancy on the course of panic disorder. Anxiety 1, 227-232 (1995).

52 Craig M, Tata P, Regan L. Psychiatric morbidity among patients with recurrent miscarriage. J. Psychosom. Obstet. Gynec.
23(3), 157-164 (2002).

53 Fertl KI, Bergner A, Beyer R, Burghard F, Klapp BF, Rauchfuss M. Levels and effects of different forms of anxiety during pregnancy after a prior miscarriage. Eur. J. Obstet. Gynecol. Reprod. Biol. 142(1) 23-29 (2009).

- Detailed discussion of the effects of adverse psychological states in pregnancy on outcome.

54 Rowlands I. Coping with miscarriage: Australian women's experiences (PhD thesis). University of Queensland, Australia (2008).

55 Rosenfeld J. Bereavement and grieving after spontaneous abortion. Am. Fam. Physician 43, 1679-1684 (1991).

56 Beutel M, Deckardt R, von Rad M et al. Grief and depression after miscarriage: their separation, antecedents and course. Psychosom. Med. 57, 517-526 (1995).

57 Herz E. Psychological repercussions of pregnancy loss. Psychiatr. Annals 14, 454-457 (1984).

58 Neugebauer R, Kline J, O'Connor P et al. Depressive symptoms in women in the six months after miscarriage. Am. J. Obstet. Gynecol. 166, 104-109 (1992).

59 Thapar AK, Thapar A. Psychological sequalae of miscarriage: a controlled study using the general health questionnaire and hospital anxiety and depression scale. Brit. J. General Practice 42, 94-96 (1992).

60 Prettyman RJ, Cordle CJ, Cook GD. A three month follow up of psychological morbidity after early miscarriage. Brit. J. Medical Psychol. 66, 363-372 (1993).

61 Goldbach KR, Dunn DS, Toedter LJ, Lasker JN. The effects of gestational age and gender on grief after pregnancy loss. Am. J. Orthopsychiatry 61, 461-467 (1991).

62 Toedtker Li, Lasker JN, Janssen HJ. International comparison of studies using the Perinatal Grief Scale: a decade of research on Pregnancy loss. Death Stud. 25, 205-238 (2001).

63 Klock S, Chang G, Hiley A, Hill J. Psychological distress among women with recurrent spontaneous abortion. Psychosomatics 38, 503-507 (1997).

64 Roswell E, Jongman G, Kilby M, Kirchmeier R, Orford J. The psychological impact of recurrent miscarriage and the role of counselling at a pre pregnancy counselling clinic. J. Reprod. Infant Psychol. 19, 33-45 (2001).

65 Johnson MP, Baker SR. Implications of coping repertoire as predictors of men's stress, anxiety and depression following pregnancy, childbirth and miscarriage: a longitudinal study. J. Psychosom. Obstet. Gynecol. 25(2) 87-98 (2004).

66 Beutel M, Wilner H, Deckardt R, von $\operatorname{Rad}$ M, Weiner H. Similarities and differences in couples' grief reactions following miscarriage: results from a longitudinal study. J. Psychosom. Res. 40, 245-253 (1996).

67 Daly S, Harte L. Does miscarriage affect the father. J. Obst. Gynecol. 16, 260-261 (1996).

68 Stinson K, Lasker J, Lohman J et al. Parent's grief following pregnancy loss: a comparison of mothers and fathers. Fam. Relat. 41, 218-223 (1992).

69 Johnson M, Puddifoot J. The grief response in the patterns of women who miscarry. Brit. J. Med. Psychol. 69, 313-327 (1996).

70 McGreal D, Evans BJ, Burrows GD. Gender differences in coping forrowing loss of a child through miscarriage or stillbirth: a pilot study. Stress Med. 13(3) 159-165 (1997).

71 Cowles K. Cultural perspectives on grief: an expanded concept analysis. J. $A d v$. Nursing 23, 287-294 (1996).

72 Hsu M, Tseng Y, Kuo L. Transforming loss: Taiwanese women's adaptation to stillbirth. J. Adv. Nursing 40, 387-395 (2002).

73 Van P, Meleis A. Coping with grief after involuntary pregnancy loss: perspectives of African-American women. J. Obstet. Gynecol. Neonatal Nurs. 32, 28-39 (2003).

74 Ventura SJ, Taffel SM. Childbearing characteristics of US and foreign born Hispanic mothers. Public Health Rep. 100, 647-652 (1985).

75 Shiono PH, Klebanoff MA, Graubard BI, Bernedes HW, Rhoads GG. Birthweight among women of different ethnic group. JAMA 225, 48-52 (1986).

76 Sefton M. Grief analysis of adolescents experiencing an early miscarriage. Hispanic Health Care Internat. 5(1) 13-20 (2007).

77 Corbet-Owen C. Women's perceptions of partner support in the context of pregnancy loss. South African J. Psychol. 33(1), 19-27 (2003).

78 Abboud N, Liamputtong P. Pregnancy loss: what it means to women who miscarry and their partners. Soc. Work Health Care 36(3), 37-62 (2003).

79 Maconochie N, Doyle P, Prior S. The National Women's Health Study: assembly and description of a population 
based reproductive cohort. BMC Pub. Health 4(1), 35 (2004).

80 Simmons R, Singh G, Maconochie N, Doyle P, Green J. Experience of miscarriage in the UK: qualitative findings from the National Women's Health Study. Soc. Sci. Med. 63, 1934-1946 (2006).

81 Bergner A, Beyer R, Klapp BF, Rauchfub M. Pregnancy after early pregnancy loss: a prospective study of anxiety depressive symptomatology and coping. J. Psychosom. Obstet. Gynecol. 28, 1-9 (2007).

82 Friedman T, Gath D. The psychiatric consequences of spontaneous abortion. Brit. J. Psychiat. 155, 810-813 (1989).

83 Forrest G, Standish E, Baum J. Support after perinatal death: a study of support and counselling after perinatal bereavement. BMJ 285, 1375-1379 (1982).

84 Cuisinier M, Kuijpers J, Hoogduin C et al. Miscarriage and stillbirth: time since the loss, grief intensity and satisfaction with care. Euro. J. Obstet. Gynecol. Reprod. Biol. 52, 163-168 (1993).

85 Lee D, Wong C, Cheung L, Leung H, Haines C, Chung T. Psychiatric morbidity following miscarriage: a prevalence study of Chinese women in Hong Kong. J. Affective Disord. 43, 63-68 (1997).

86 Goff B, Smith D. Systemic traumatic stress: the couple adaptation to traumatic stress model. J. Marital Fam. Ther. 31, 145-157 (2005).

87 Goldberg D. General Health Questionnaire (GHQ-12). Windsor, UK: NFER-Nelson (1992).

88 Zigmond AS, Snaith RP. The Hospital Anxiety and Depression Scale. Acta Psychiat. Scand. 67, 361-370 (1983).

89 Nikcevic A, Tunkel S, Nicolaides K. Psychological outcomes following missed abortions and provision of follow up care. Ultrasound Obstet. Gynecol. 11, 123-128 (1998).

90 Cecil R. Miscarriage: women's views of care. J. Reprod. Infant Psychol. 12, 21-30 (1994).
91 Brier N. Understanding and managing the emotional reactions to miscarriage. Obstet. Gynecol. 93, 151-155 (1999).

92 Moohan J, Ashe RG, Cecil R. The management of miscarriage: results from a survey at one hospital. J. Reprod. Infant Psychol. 12, 17-19, (1994).

93 Cecil R. Miscarriage: women's views of care. J. Reprod. Infant Psychol. 12, 21-29 (1994).

94 Cordle CJ, Prettyman RJ. A 2 year follow up of women who have experienced early miscarriage. J. Reprod. Infant Psychol. 12, 37-43 (1994).

95 Sejourn N, Callahan S, Chabrol H. Support following miscarriage: what women want. J. Reprod. Infant Psychol. 28(4), 403-411 (2010).

96 Stratton K, Lloyd L. Hospital based interventions, at and following miscarriage: literature to inform a research-practice initiative. Aust. NZ J. Obstet. Gynaecol. 48(1), 5-11 (2008).

97 Geller PA, Psaros C, Kornfield SL. Satisfaction with pregnancy loss aftercare: are women getting what they want? Arch. Womens Mental Health 13, 111-124 (2010).

98 Weiss L, Frischer L, Rickman J. Parental adjustment to intrapartum and delivery room loss: the role of a hospital-based support programme. Clin. Perinatol. 16, 1009-1019 (1989).

99 Swanson KM. Effects of caring, measurement and time on miscarriage impact and women's well being. Nursing Res. 48(6) 288-298 (1999).

100 Neugebauer R, Kline J, Markowitz J et al. Pilot randomized controlled trial of interpersonal counseling for subsyndromal depression following miscarriage. J. Clin. Psychiatry 67, 1299-1304 (2006).

101 Moulder C. Miscarriage: Women's Experiences and Needs. Routledge, London, UK (2001).

102 Wong M, Crawford T, Gask L, Grinyer A. A qualitative investigation into women's experiences after a miscarriage: implications for the primary healthcare team. Brit. J. Gen. Prac. 53(494), 697-702 (2003).
103 Simmons RK, Singh G, Maconochie N, Doyle P, Green J. Experience of miscarriage in the UK: qualitative findings from the National Women's Health Study. Soc. Sci. Med. 63(7) 1934-1946 (2006).

- Describes qualitatively the needs of miscarrying women, emphasizing the individual woman's concerns about medicalization of the event.

104 Logan S, Browne J, Bhattacharya S. More Units dedicated to women presenting with miscarriage are needed. BMJ 324(7352), 1526 (2002).

105 RCOG. Early Pregnancy Loss, Management (Green-top 25) Guidelines. London, UK (2000).

106 Boyce P, Condon J, Ellwood D. Pregnancy loss: a major life event affecting emotional health and wellbeing. Med. J. Australia 176(6), 250-251 (2002).

107 Wang V, Marsh FH. Ethical principles and cultural integrity in health care delivery: Asian ethnocultural perspectives in genetic services. J. Genetic Couns. 1, 81-92 (1992).

108 Sue DW, Sue D. Counseling The Culturally Different: Theory and Practice (2nd Edition). John Wiley and Sons, NY, USA (1999).

109 van der Sijpt E. Marginal matters: Pregnancy loss as a social event. Soc. Sci. Med. 71, 1773-1779 (2010).

- Outlines the shift in thinking about demographic factors affecting reproductive health. The author shows how traditional concerns in developing countries reproductive health are now considering reproductive rights, choices and stigma.

\section{Website}

201 RCOG Guideline No. 178 of 13 www.rcog.org.uk/womens-health/ clinical-guidance/couples-recurrentmiscarriage-what-rcog-guideline-meansyou. 